\title{
HIERARCHICALLY DECOMPOSED FINITE ELEMENT METHOD FOR THE COUPLED FOUR FIELDS OF THE FLUID-STRUCTURE-PIEZOELECTRIC-CIRCUIT INTERACTION
}

\author{
Prakasha Chigahalli Ramegowda*, Daisuke Ishihara ${ }^{\dagger}$ \\ ${ }^{*}$ Department of Intelligent and Control Systems, \\ Kyushu Institute of Technology, 680-4 Kawazu, Iizuka, Fukuoka, 820-8502, Japan \\ e-mail: prakasha@solid.mse.kyutech.ac.jp
}

Key words: Fluid-structure-piezoelectric-circuit interaction, hierarchal decomposition, Partitioned iterative method, Finite element method

\begin{abstract}
A partitioned iterative method based on hierarchical decomposition is proposed for providing numerical modeling and analysis of the piezoelectric energy harvester which is involved in coupled fluid-structure interaction, coupled electro-mechanical, and a controlling electrical circuit for piezoelectric structural applications in energy harvesting. This circuitintegrated piezoelectric structural application in energy harvesting surrounded by fluid media takes the form of natural four-way coupling of fluid flow, the structure, the electromechanical effect of the piezoelectric material, and the electrical circuit. This can be formulated exactly as a fluid-structure-piezoelectric-circuit interaction. These coupled four fields are hierarchically decomposed into the fluid-structure interaction, structure-piezoelectric interaction, and piezoelectric-circuit interaction interactions. Then these subsystems are decomposed into each field. The proposed finite element method enables to reuse of existing techniques because of its modularity. Furthermore, scalability to multiphysics and multisystem couplings is expected. There are some numerical approaches in particular monolithic coupling methods are studied which are computationally expensive and leads to an ill-conditioned coefficient matrix. Nevertheless, accurate modeling for predicting the characteristics of this four-way coupling using partitioned methods has not yet been developed. This method enables an investigation of piezoelectric structures in fluid with complex geometry, material composition, and attached electrical circuits to the harvester. A flexible piezoelectric bimorph harvester in the converging channel is analyzed to demonstrate the efficiency of the proposed method. The results indicate that the method captures the coupled effect accurately.
\end{abstract}

\section{Introduction}

The circuit-integrated piezoelectric devices surrounded by fluid media take the form of natural four-way coupling of fluid flow, the structure, the electromechanical effect of the piezo- 


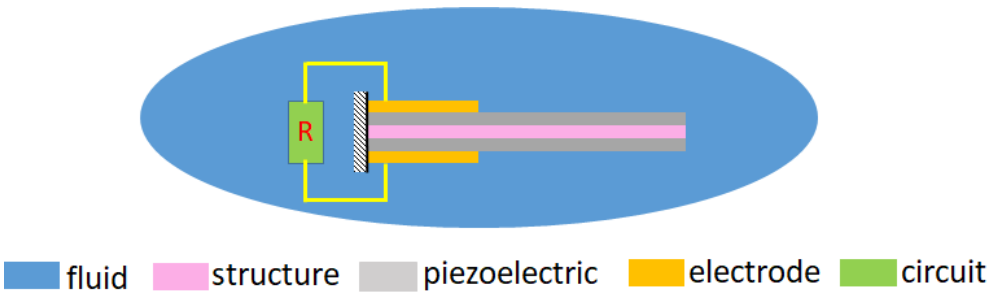

Figure 1: Subdomains of the multiphysics coupled problem

electric material, and the electrical circuit. This type of multiphysics coupling is a common phenomenon that appears in flow-induced piezoelectric energy harvesting [1,2]. As shown in Fig. 1, this piezoelectric energy harvesting technology simultaneously involves the coupled interaction of a composite piezoelectric structure and a surrounding fluid, the electric charge stored in the piezoelectric material, and a controlling electrical circuit attached to the harvester. The large deformation of a thin piezoelectric device by the fluid flow causes a strong interaction with the electric field and the surrounding fluid, and inversely, these two fields significantly affect the structural behavior. Also, the electrical charge in the circuit affects the electromechanical behavior of the piezoelectric oscillator because of the coupled direct-piezoelectric and inverse-piezoelectric effects. At the same time, the piezoelectric device behaves as a kind of ca-

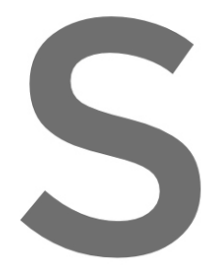
pacitance for the circulit, which affects the elect
exactly as a fluid-structure-piezoelectric-circuit
of such smart future devices and to increase th
model of the complex physical system is requited
of the complex phenomena and multiphysics coup
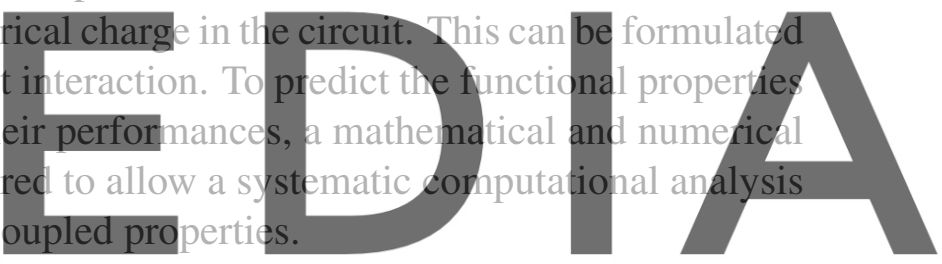

On the mathematical modeling front, Amini et al. [3] recently proposed a numerical model

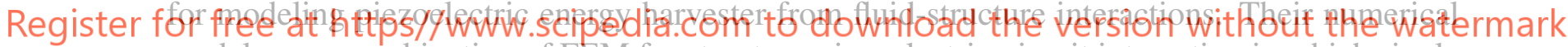
model was a combination of FEM for structure-piezoelectric-circuit interaction in which single degree of freedom (SDOF) is used for electric circuit and open FOAM solver for fluid. In their finite element models considered a linear variation of electrical potential through-the-thickness of the piezoelectric continuum. Several research works [4-12] clearly pointed out that the electromechnical coupling is partial in case of a linear approximation. Yang [13], Klinkel and Wagner [14] was demonstrated that linear approximation was incorrect. Further, Yang [13] investigated and shown that a quadratic approximation of electric potential through-the-thickness of the piezoelectric continuum is necessary in bending-related problems. Akaydin et al [15]. analyzed energy harvesting from unsteady turbulent flow by placing a piezoelectric beam in the wake of a circular cylinder at high Reynolds number. They used commercial FLUENT software to analyze the coupled interactions of the aerodynamics of turbulent flow, the piezoelectric structure under vibration, the electrical response of the piezoelectric material and the harvester electronic circuit. They used SDOF model for the piezoelectric beam. Both the piezoelectric structure and electric circuit governing equations are reduced to a second-order- ordinary differential equations. Erturk et al [16] investigated that SDOF model may yield highly inaccurate 


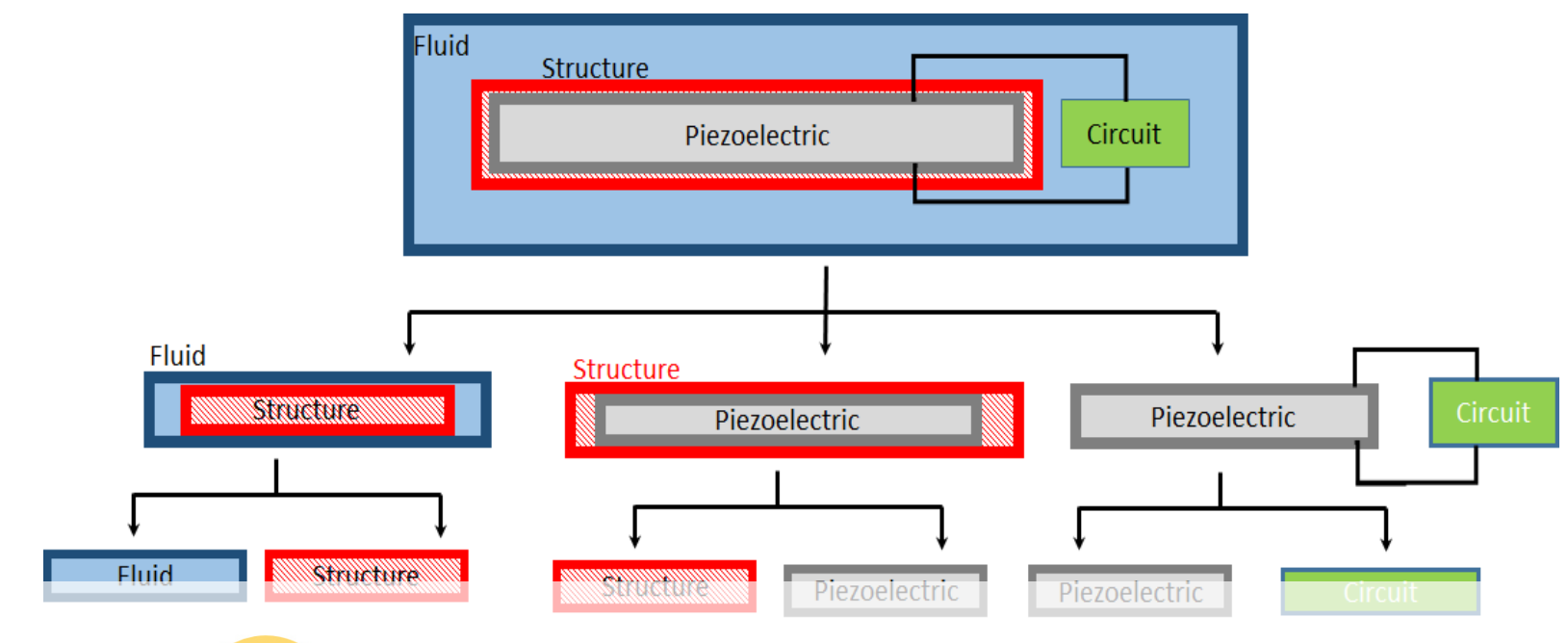

Figure 2: Hierarchical decomposition of the fluid-structure-piezoelectric-circuit interaction .

results for transverse vibrations of piezoelectric beams and can not accurately predict the power outputs of the harvester. Very recently, Ravi et al. [17] had proposed simultaneous solution method also called as monolithic method wherein the fluid, the piezoelectric structure with

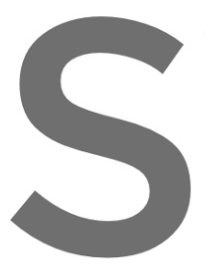
electrode, and the eldet ic simultaneously using $\mathrm{F}$ strongly coupled, and 20]. Nevertheless, this software modularity is difficult with monolithi
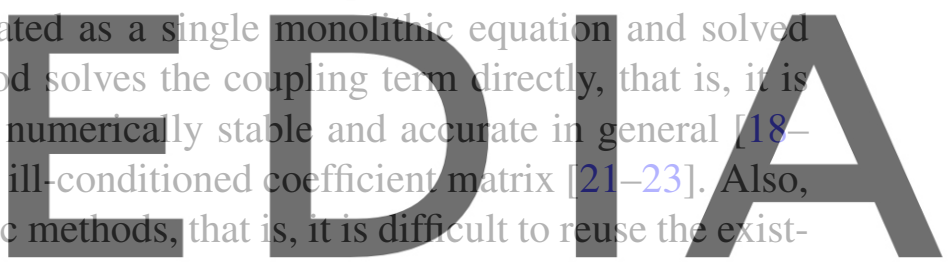
ing technologies developed in different domains, and ad hoc software development is required

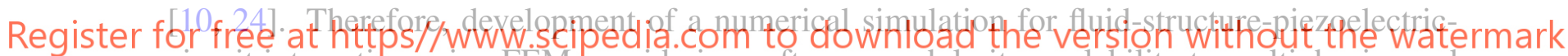
circuit interaction using FEM considering software modularity, scalability to multiphysics and multisystem coupling, strong coupling, and solution accuracy is very important. This can be expected using a partitioned iterative coupled algorithms [25-29].

In the proposed method, the fluid-structure-piezoelectric-circuit interaction system is decomposed into subsystems hierarchically. This system is decomposed into the fluid-structure interaction (FSI), structure-piezoelectric interaction, and piezoelectric-circuit interaction using a iterative algorithm is similar to the partitioning of structure-piezoelectric-circuit interaction [30, 31], structure-piezoelectric interaction [11, 12], fluid-structure-piezoelectric interaction [25], and fluid-structure-electrostatic interaction[26]. This type of framework can be characterized as hierarchical decomposition. Here, the FSI subsystem is further split into the fluid-structure velocity field and the pressure field using the projection method [32]. Then, the structure-fluid velocity field is further partitioned into the structure velocity field and the fluid velocity field using the explicit time integration for the fluid. The structure-piezoelectric interaction is decomposed into the structure field (inverse-piezoelectric field) and the directpiezoelectric field. Similarly, the piezoelectric-circuit interaction system is decomposed into 
the direct-piezoelectric field and the circuit. In essence, the fluid-structure-piezoelectric-circuit interaction system is decomposed into each field of four distinct fluid, structure, direct piezoelectric, and circuit solvers, as shown in Fig. 2. A thin flexible piezoelectric bimorph harvester in a converging channel is solved using the proposed method. Results demonstrated that the proposed method is accurate analyzes coupling phenomena.

\section{Strong-form governing equations}

The complete set of mathematical model equations of the strong-form governing equations of the coupled problem of the fluid, structure, piezoelectric material connected to the electrodes, and the electrical circuit is given here. Eqs. (1)-(4) represents the Navier-Stokes equations for an incompressible fluid. The arbitrary Laqrangian-Eulerian formulation is employed.

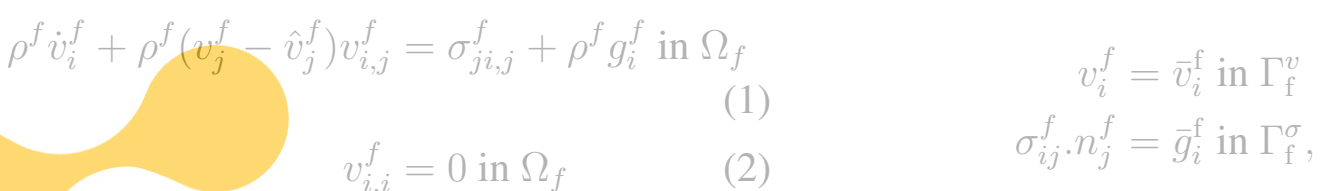

Eqs. (5)-(9) represents the inverse piezoelectric effect of the piezoelectric material. Thesed equations also represents elastic structure if piezoelectric constant tensor $e_{i j k}$ is zero. In the domain occupied by the piezoelectric material, the present electric field representing the directpiezoelectric effect is tive relations (6) and (1 direct-piezoelectric interaction
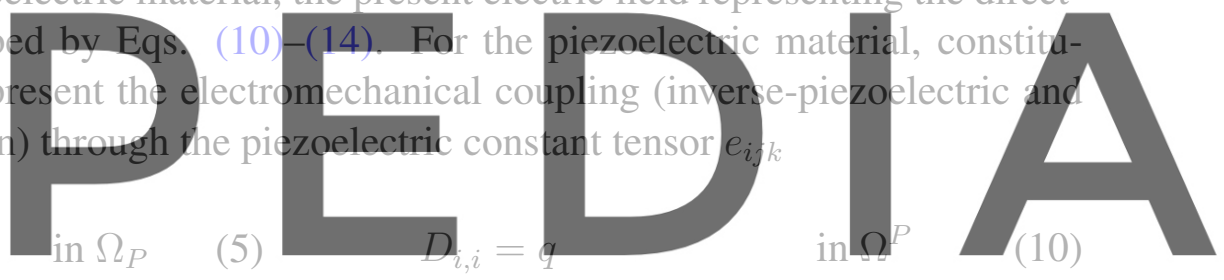

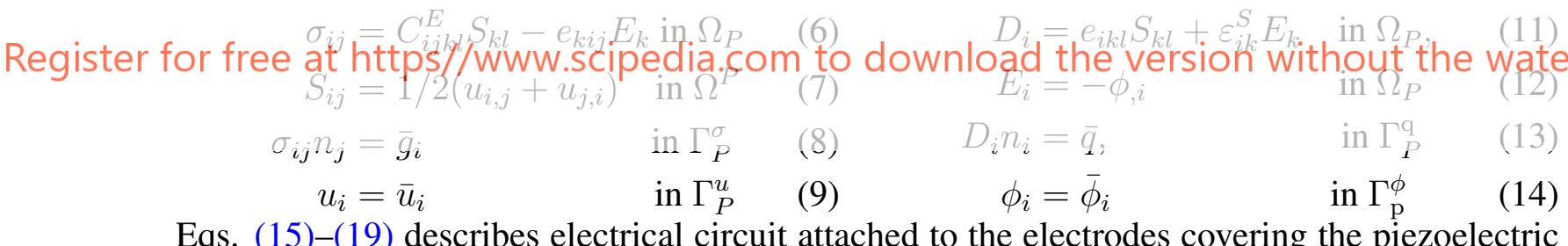
material. Eq.(15) represents the electrical circuit described using Kirchhoff's lawwith load resistance $R$. Eq.(19) represents the equi-potential condition on the electrode making use of the surface boundary charge $\bar{q}$ in (13). Eqs.(19) and (30) are the potential continuity and charge continuity, respectively, representing a native coupling between circuit and the electrode.

$$
\begin{aligned}
R \dot{Q}+V_{p} & =V_{c} & & \text { in } \Gamma_{e} \\
\bar{\phi}_{+}-\phi & =0 & & \text { in } \Gamma_{e}^{+} \\
\bar{\phi}_{-}-\phi & =0 & & \text { in } \Gamma_{e}^{-}
\end{aligned}
$$

$$
\begin{aligned}
& V_{p}=\bar{\phi}_{+}-\bar{\phi}_{-} . \quad \text { in } \Gamma_{P}^{e} \\
& Q=\int_{\Gamma_{e}^{+}} q_{+} \mathrm{d} \Gamma_{\mathrm{e}}=-\int_{\Gamma_{e}^{+}} q_{+} \mathrm{d} \Gamma_{\mathrm{e}}
\end{aligned}
$$

The interaction conditions on the interface between the fluid and the piezoelectric structure are imposed using the following geometric compatibility (20) and equilibrium conditions (21): 


$$
v_{i}^{\mathrm{f}}=v_{i} \equiv v_{i}^{\mathrm{fs}}, \quad \text { on } \Gamma_{\mathrm{fs}},
$$

$$
\sigma_{i j}^{\mathrm{f}} \cdot n_{j}^{\mathrm{f}}+\sigma_{i j} \cdot n_{j}=\bar{g}_{i}^{\mathrm{f} s}, \quad \text { on } \Gamma_{\mathrm{fs}},
$$

\section{Finite element equations and coupling strategy}

The weak form involving the unknown variables of fluid velocity $\mathbf{v}^{\mathbf{f}}$, fluid pressure $\mathbf{P}^{\mathbf{f}}$, piezoelectric structure velocity $\mathbf{v}$, the electrical potential with in the piezoelectric material $\phi$, and the total circuit charge $Q$ is obtained using the weighted residual method for the above set of strong form governing equations. Employing the standard finite element discretization procedure leads to an algebraic set of equations and using heirarchical decomposition and partitioned iterative solver for system of algebraic equations one obtain a solution method for the multiphysics problem. Eqs. (22)-(25) represents the finite element spatial discretization to the incompressible Navier-Stokes Eqs. (1)-(4). Eqs.(26) and (27) represents the FE coupled inverse-piezoelectric and direct-piezoelectric effect of a piezoelectric material. The pure structural part of this coupled system is obtained using Eq.(26) with $\mathbf{K}_{\mathrm{u} \phi} \phi=0$ shown in Eq.(28). Similarly the electrodes are analyzed using pseudo-piezoelectric method leading to Eqs.(28) and (29) [12, 33, 34],

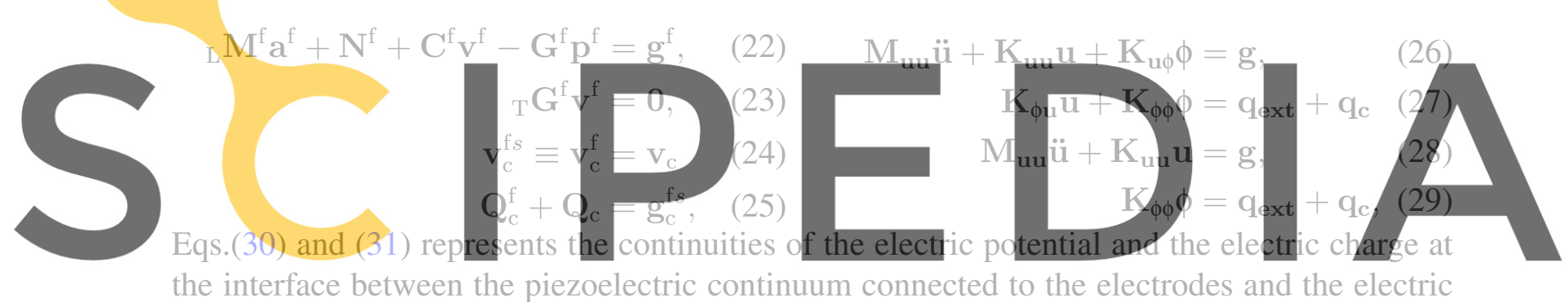

Register foirquiee at https//www.scipedia.com to download the version without the watermark

$$
\mathbf{V}_{\mathbf{p}}=\bar{\phi}_{+}-\bar{\phi}_{-} \quad \text { (30) } \quad \mathbf{q}_{\mathbf{c}}=\int_{\mathrm{S}^{\mathrm{c}}} \mathrm{N}_{\phi}\left(\mathrm{Q} / \mathrm{S}^{\mathrm{c}}\right) d S-\int_{\mathrm{S}_{-}^{\mathrm{c}}} \mathrm{N}_{\phi}\left(\mathrm{Q} / \mathrm{S}_{-}^{\mathrm{c}}\right) d S
$$

where $\mathbf{M}^{\mathrm{f}}$ is the mass matrix of the fluid, $\mathbf{N}^{f}$ is the convective term vector of the fluid, $\mathbf{C}^{\mathrm{f}}$ is the diffusion matrix of the fluid, $G^{f}$ is the divergence operator matrix of the fluid, $\mathbf{a}^{f}$ is the acceleration vector of the fluid, $\mathbf{v}^{\mathrm{f}}$ is the velocity vector of the fluid, $\mathbf{p}^{\mathrm{f}}$ is the pressure vector of the fluid, $\mathrm{g}^{\mathrm{f}}$ is the external force vector acting on the fluid, $\mathrm{Q}^{\mathrm{f}}$ is the equivalent internal force vector including all effects of the fluid, $\mathrm{Q}$ is the equivalent internal force vector including all effects of the piezoelectric structure, the subscript L stands for lumping of the matrix, and the subscript $\mathrm{T}$ stands for transpose of the matrix. In the ALE formulation, the fluid convective term $\mathbf{N}^{f}$ is expressed as $\mathbf{N}^{\mathrm{f}}\left(\mathbf{v}^{\mathrm{f}}-\hat{\mathbf{v}}^{\mathrm{f}}\right) \mathbf{v}^{\mathrm{f}}$, where $\mathbf{M}_{u} u$ is the mass matrix of the piezoelectric structure, $\mathbf{K}$ is the mechanical stiffness matrix of the piezoelectric material, $\mathbf{K}_{\phi \phi}$ is the dielectric stiffness matrix of the piezoelectric material, $\mathbf{K}_{\mathrm{u} \phi}$ is the piezoelectric coupling coefficient matrix of the piezoelectric material, $\mathbf{u}$ is the vector of the mechanical displacements, $\phi$ is the vector of electric potentials, $g$ is the vector of the external mechanical forces, qext represents 


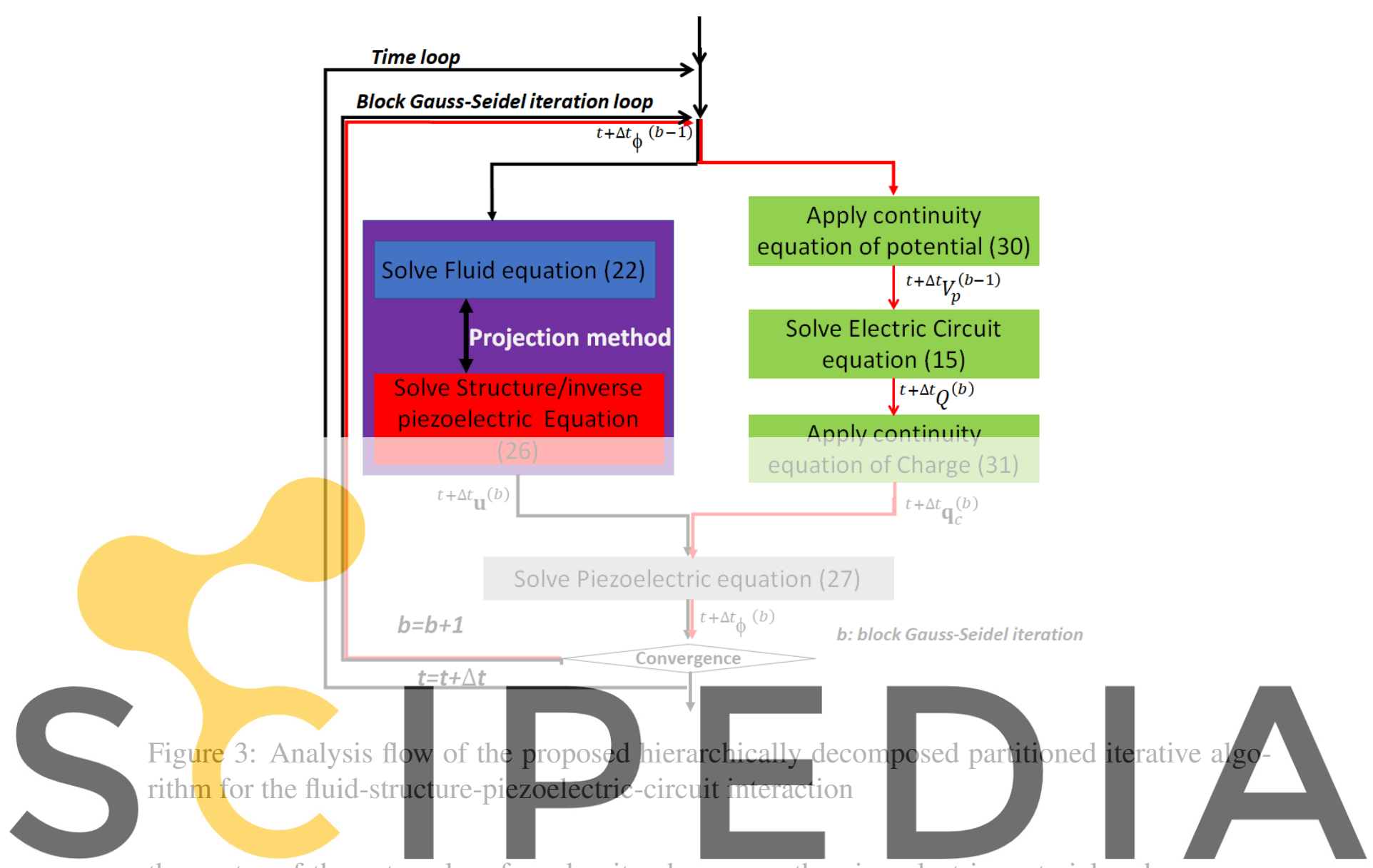

the vector of the external surface density charges on the piezoelectric material and $\mathrm{q}_{\mathrm{c}}$ repre-

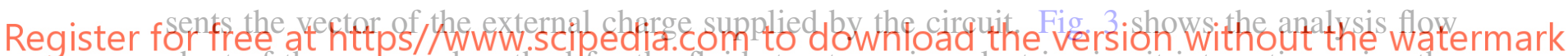
chart of the proposed method for the fluid-structure-piezoelectric-circuit interaction using the

partitioned iterative algorithm. The projection method [32] using the algebraic splitting is used

to solve the fluid-structure interaction. And finally, all the fields are coupled using loop union through Block Gauss-Seidel iterative procedure, as shown in Fig. 3.

\section{Numerical example: A thin flexible piezoelectric bimorph harvester in a converging channel}

\subsection{Problem setup}

A flexible piezoelectric bimorph harvester in the converging channel is analyzed in order to demonstrate the coupling between fluid, piezoelectric structure, and the circuit. The fulud domain is modeled using stabilized linear equal-order-interpolation velocity-pressure elements [35] (2,982 nodes and 8,400 elements), the structural part of the piezoelectric structure is modeled using mixed interpolation of the tensoral components shell elements [36] (22 nodes and 10 elements), while the electrical part of the piezoelectric bimorph is modeled using 20 node hexahedron solid elements (503 nodes and 60 elements). The fluid is air and the bimorph is made of 


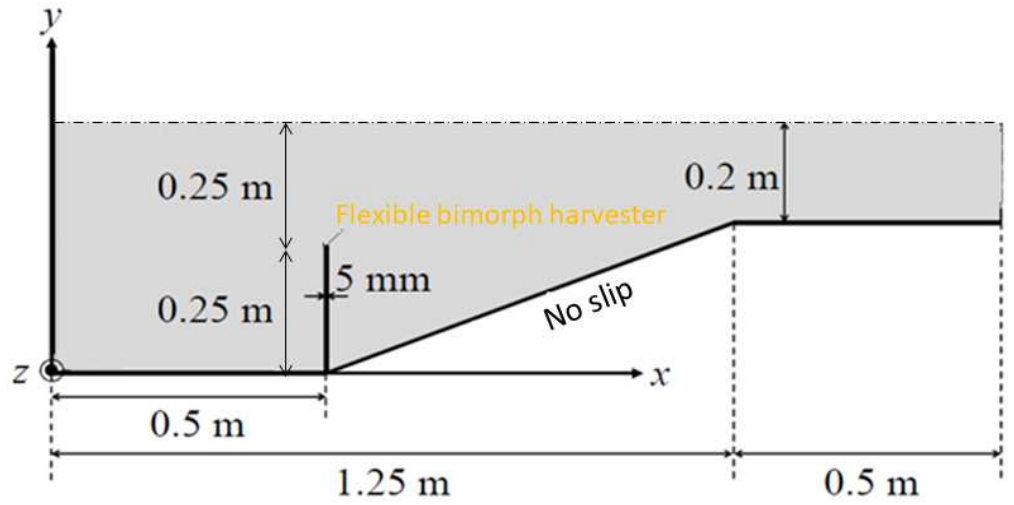

(a) Flexible piezoelectric bimorph energy harvester in fluid channel

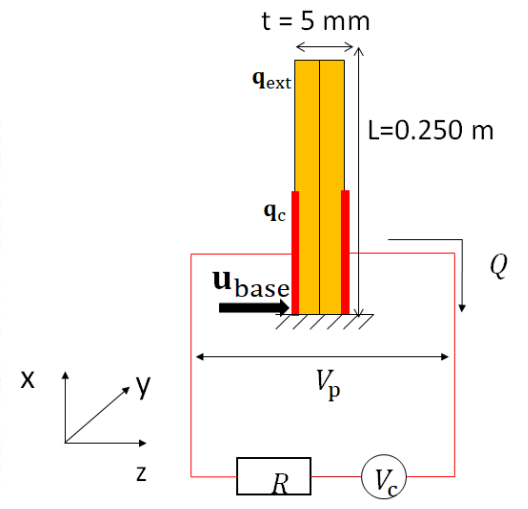

(b) Close view of harvester in fluid.

Figure 4: A flexible piezoelectric bimorph energy harvester in a converging fluid channel subjected to mechanical base excitation $\mathbf{u}_{\text {base }}$ and attached to an circuit with load resistance $R$.

PZT-5A material. The base acceleration of level is set as $a_{0}=9.81 \mathrm{~m} / \mathrm{s}^{2}$. The forced displacement at the fixed end of the bimorph $\mathrm{x}=0$ as a function of time $t$ is given as $u_{\text {base }}=u_{0} \sin \omega t$, as shown in Fig. 4. The forced acceleration amplitude $a_{0}$ and the forced displacement amplitude

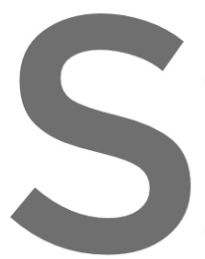
are related through $u$ electrical resistance is study short circuit condition. $5.0 \times 10^{-5}$ sec for shor cuit [30] and the Courant numbe

length $\mathrm{L}=250 \mathrm{~mm}$, width $\mathrm{w}=20 \mathrm{~mm}$, and $\mathrm{t}=2 \mathrm{t}_{\mathrm{p}}=5.0 \mathrm{~mm}$. The variables from the shell

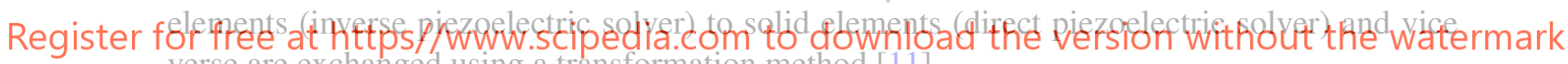
verse are exchanged using a transformation method [11].

\subsection{Results and discussions}

Figures 5(a) and 5(b) shows the time histories of the tip deflection of the bimorph harvester when excited at $f=40.0 \mathrm{~Hz}$ (near resonance) and the fluid velocities at the fluid node attached to the tip of the bimorph, respectively. As shown in these figures, we can see a beat phenomena due to the resonance. The method can capture the resonance characteristic accurately. Fig. 6(a) shows the time histories of the generated voltages due to the piezoelectric effect flowing through the attached circuit at load resistance $\mathrm{R}=1.0 \mathrm{M} \Omega$ (black curve: open circuit condition) and $\mathrm{R}=1.0 \mathrm{~K} \Omega$ (red curve: short circuit condition). As shown in this figure, in the case of short circuit configuration the voltage is very very less (close to zero) than that of the open circuit condition because of the no piezoelectric effect, and therefore, no resistance is offered, hence, the current is maximum in the circuit, and vice-versa, that is, the generated voltage is maximum at open circuit condition, as shown in Fig. 6(b). The simulation results follow the standard electric circuit theory. 


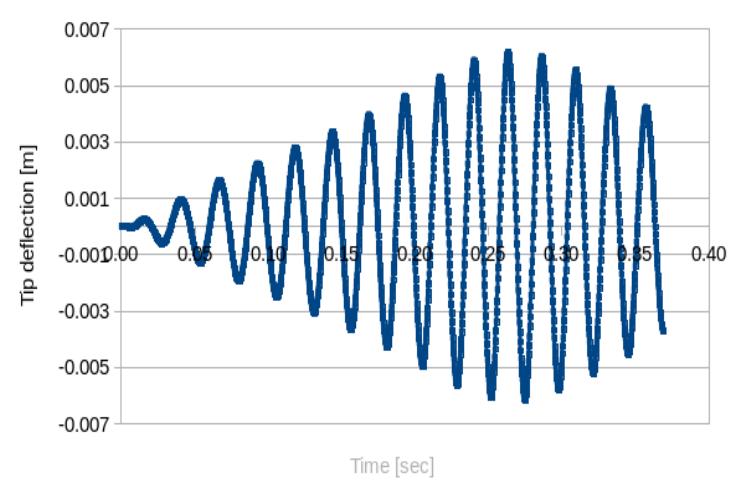

(a)

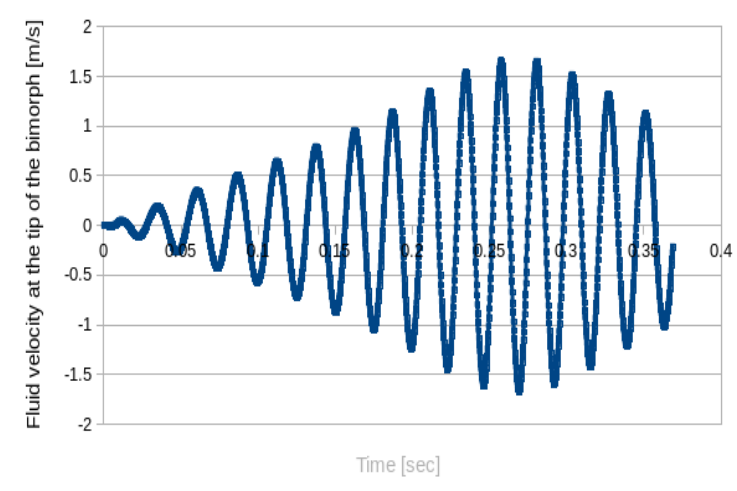

(b)

Figure 5: Time histories of tip deflection of the bimorph (a) and the fluid velocity at the tip of the structure (b).
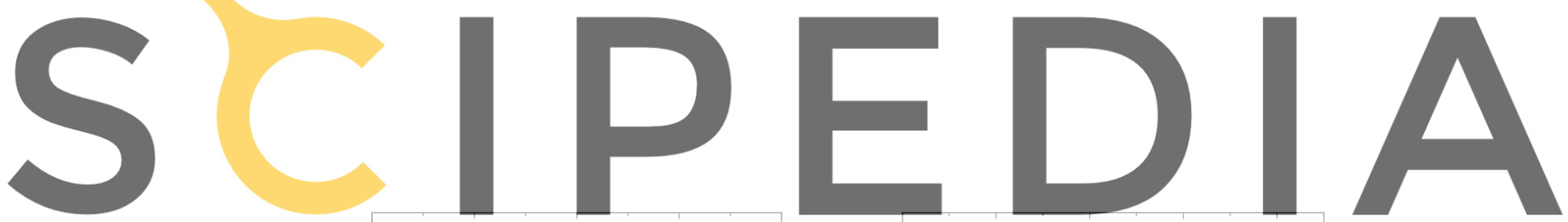

Register for free at https//Wrw. Seipedila.coln to download the erefsion without the watermark

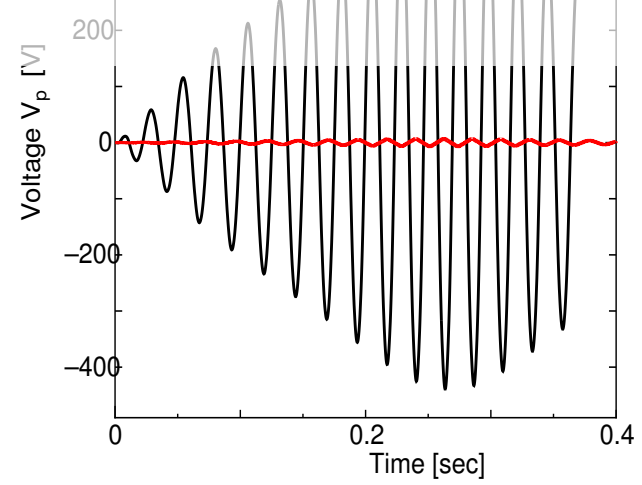

(a)

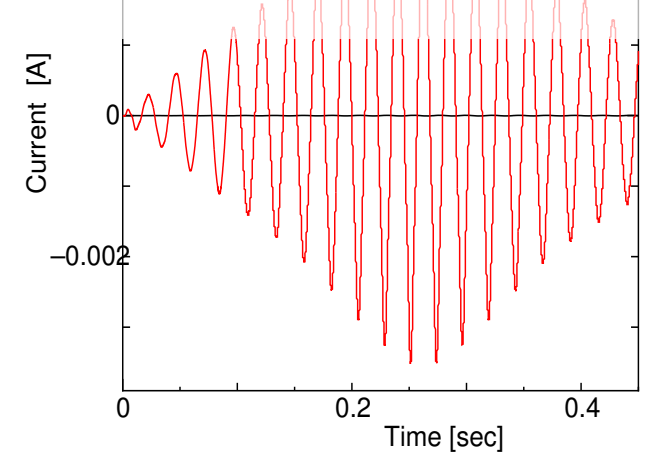

(b)

Figure 6: Time histories of generated voltage (a) and the current flowing through the circuit (b). 


\section{Concluding remarks}

In this study, a partitioned iterative method is proposed for the four-way coupling of fluid, the structure, the electromechanical effect of the piezoelectric material, and the electrical circuit based on hierarchical decomposition. These coupled four fields are hierarchically decomposed into the fluid-structure interaction, structure-piezoelectric interaction, and piezoelectric-circuit interaction interactions. Then these subsystems are decomposed into each field. Nevertheless, accurate modeling for predicting the characteristics of this four-way coupling using partitioned methods is first developed in this work. Using the proposed method, a flexible piezoelectric bimorph harvester in the converging channel is analyzed to demonstrate the coupling between fluid, piezoelectric structure, and the circuit. The results indicate that the method captures the coupled effect accurately.

\section{References}

[1] Y. Tanaka, T. Oko. H. Mutsuda, R. Patel, S.M. William, A.A. Popov, An experimental study of wave power generation using a flexible piezoelectric devices, Journal of Ocean and Wind Energy, 2 (2015) 28-36.

[2] H. Matsuda, Y. Tanaka, R. Patel, Y. Doi, , Harvesting flow-induced vibration using a
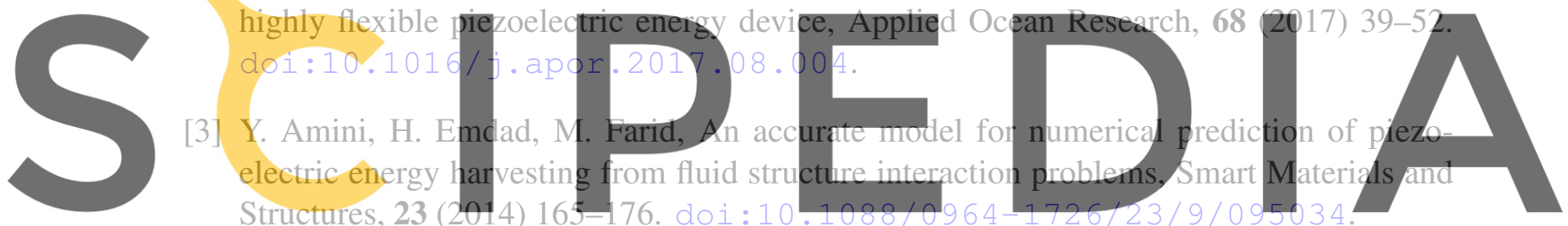

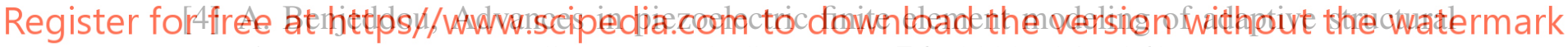
elements: a survey, Computers \& Structures 76 (2000) 347-363. doi:10.1016/ S0045-7949(99)00151-0.

[5] S.K. Ha, C. Keilers, F.K. Chang, Finite element analysis of composite structures containing distributed piezoelectric sensors and actuators, AIAA Journal 30 (1992) 772-780. doi: $10.2514 / 3.10984$.

[6] R. MacNeal, R. Harder, A proposed standard set of problems to test finite element accuracy, Finite Elements in Analysis and Design 1 (1985) 3-20. doi:10.1016/ $0168-874 \times(85) 90003-4$.

[7] I.S. KoKo, I.R. Orisamolu, M.J. Smith, U.O. Alepan, Finite element based design tool for smart composite structures, Smart Structures and Materials:SPIE 3039 (1997) 125-134.

[8] S. Ravi, A. Zilian, Monolithic modeling and finite element analysis of piezoelectric energy harvesters, Acta Mechanica, 228 (2017) 2251-2261. doi:10.1007/ s00707-017-1830-7. 
[9] S. Ravi, A. Zilian, Time and frequency domain analysis of piezoelectric energy harvesters by monolithic finite element modeling, International Journal for Numerical Methods in Engineering, 112 (2017) 1828-1847. doi : $10.1002 /$ nme. 5584.

[10] P.C. Ramegowda, D. Ishihara, T. Niho, T. Horie, Performance evaluation of numerical finite element coupled algorithms for structure-electric interaction analysis of mems piezoelectric actuator, International Journal of Computational Methods 16(7) (2019) 1850106. doi:10.1142/s0219876218501062.

[11] P.C. Ramegowda, D. Ishihara, T. Niho, T. Horie, A novel coupling algorithm for the electric field-structure interaction using a transformation method between solid and shell elements in a thin piezoelectric bimorph plate analysis, Finite Elements in Analysis and Design 159 (2019) 33-49. doi:10.1016/j. fine1.2019.02.001.

[12] P.C. Ramegowda, D. Ishihara, R. Takata, T. Niho, T. Horie, Finite element analysis of thin piezoelectric bimorph with a metal shim using solid direct-piezoelectric and shellinverse piezoelectric coupling with pseudo-piezoelectric evaluation, Composite Structures 245 (2020) 112284. doi:10.1016/j.compstruct.2020.112284.

[13] J.S. Yang, Equations for thick elastic plates with partially electroded piezoelectric actuators and higher order electric fields, Smart Materials and Stryctures 8 (1999) 73.
doi: $10.1088 / 0964-1726 / 8 / 1 / 008$.
S. Klinkel, W. Wagner, A geometrically non-linear piezoelectric so/id/shell element pased
on a mixed multi-ffeld variational formulation, International Journal for Numerical/Methods in Engineering 65 (2006) 349-382. doi:10.1002/nme.1447.

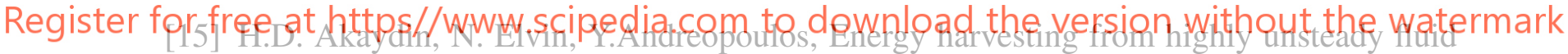
flows using piezoelectric materials, Journal of Intelligent Material Systems and Structures, 21 (2010) 1263-1278. doi:10.1177/1045389x10366317.

[16] A. Erturk, D.J. Inman, Issues in marhematical modeling of piezoelectric energy harvesters, Smart Materials and Structures, 17 (2008) 1065016. doi : 10.1088 / $0964-1726 / 17$ / $6 / 065016$.

[17] S. Ravi, A. Zilian, Simultaneous finite element analysis of circuit-integrated piezoelectric energy harvesting from fluid-structure interaction, Mechanical Systems and Signal Processing, 114 (2017) 259-274. doi:10.1016/j.ymssp.2018.05.016.

[18] T. Niho, T. Horie, J. Uefuji, D. Ishihara, Stability analysis and evaluation of staggered coupled analysis methods for electromagnetic and structural coupled finite element analysis, Computers \& Structures 178 (2017) 1129-142. doi:10.1016/ j. compstruc. 2016.09 .003$. 
[19] B. Hubner, E. Walhorn, D. Dinkler, A monolithic approach to fluid-structure interaction using space-time finite elements, Computer Methods in Applied Mechanics and Engineering, 193 (2004) 2087-2104. doi: $10.1016 /$ j.cma. 2004.01 .024$.

[20] Q. Zhang, T. Hisada, Analysis of fluid-structure interaction problems with structural buckling and large domain changes by ale finite element method, Computer Methods in Applied Mechanics and Engineering, 190 (2001) 6341-6357. doi:10.1016/ s $0045-7825$ (01) 00231-6.

[21] H. Kuramae, H. Nishioka, Y. Uetsuji, E. Nakamachi, Development and performance evaluation of parallel iterative method for multi-scale piezoelectric analyses, Transactions of the Japan Society for Computational Engineering and Science 2007 (2007) 220070033.

[22] D. Ishihara, S. Yoshimura, A monolithic approach for interaction of incompressible viscous fluid and an elastic body based on fluid pressure poisson equation, International Journal for Numerical Methods in Engineering 64 (2005) 167-203. doi:10 . 1002 / nme. 1348.

[23] S. Rugonyi, K. J. Bathe, On finite element analysis of fluid flows fully coupled with structural interactions, Computer Modeling in Engineering and Sciences 2 (2001) 195-212.

[24] S. Badia, A. Quaini, A. Quarteron, Splitting methods based on algebraic factorization for fluid-structure interaction,, SIAM Journal on Scientific Computing 30 (2008) 1778-1805. doi:10.1137/070680497.

[25] P.C. Ramegowda, D. Ishihara, R. Takata, T. Niho, T. Horie, Hierarchically decomposed finite element method for a triply coupled piezoelectric, structure, and fluid fields of a thin piezoelectric bimorph in fluid, Computer Methods in Applied Mechanics and Engineering 365 (2020) 113006. doi:10.1016/j.cma.2020.113006.

[26] D. Ishihara, T. Horie, T. Niho, T. Baba, Hierarchal decomposition for the structure-fluidelectrostatic interaction in a microelectromechanical system., Computer Modeling in Engineering and Sciences. 108 (2015) :429-452.

[27] C.A. Felippa, K.C. Park, C. Farhat, Partitioned analysis of coupled mechanical systems,, Computer Methods in Applied Mechanics and Engineering 190 (2001) 3247-3270. do i : $10.1016 / \mathrm{s} 0045-7825$ (00)00391-1.

[28] Ramegowda, P.C. Ishihara, D. Takata, R. Niho,T. and Horie, T, A finite element approach for a coupled numerical simulation of fluid -structure-electric interaction in mems., Proceeding of the VII International Conference Computational Methods for Coupled Problems In Science and Engineering, Greece. (2017) :999-1007. 
[29] Ramegowda, P.C. Ishihara, D. Takata, R. Niho,T. and Horie, T, Fluid-structure and electric interaction analysis of piezoelectric flap in a channel using a strongly coupled fem scheme, Proceeding of the 6th European Conference on Computational Mechanics (ECCM 6 2018), Glasgow. (2018) :382-393.

[30] D.Ishihara, R. Takata, P.C. Ramegowda, N. Takayama, Strongly coupled partitioned iterative method for the structure-piezoelectric-circuit interaction using hierarchical decomposition, Computers \& Structures 253 (2021) 106572. doi:10.1016/j.compstruc. 2021.106572 .

[31] D.Ishihara, R. Takata, P.C. Ramegowda, Partitioned iterative method for inverse piezoelectricdirect piezoelectricelectric circuit interaction, Transactions of the Japan Society for Computational Engineering and Science 2020 (2020) 20200015. doi : 10 . $11421 /$ jsces.2020.20200015.

[32] D.Ishihara, T. Horie, A projection method for the monolithic interaction system of an incompressible fluid and a structure using a new algebraic splitting., Computer Modeling in Engineering and Sciences. 101 (2014) :421-440.

[33] R. Takata,D.Ishihara, P.C. Ramegowda, T. Niho, T. Horie, Solid piezoelectric - shell inverse piezoelectric partitioned analysis method for thin piezoelectric bimorph with conductor layers, Transactions of the Japan Society for Computational Engineering and Science 2019 (2019) 20190011. doi : 10 . 11421/ jsces . 2019.20190011.

[34] D. Ishihara, P.C. Ramegowda, R. Takata, T. Niho, T. Horie, Coupled solid piezoelectric and shell inverse-piezoelectric analysis using partitioned method for thin piezoelectric bimorph with metal layers., Proceeding of the VIII International Conference Computational Methods for Coupled Problems In Science and Engineering. Spain (2019) :451-457.

[35] T.E. Tezduyar, S. Mittal, S. Ray, R. Shih, Incompressible flow computations with stabilized bilinear and linear equal-order-interpolation velocity-pressure elements., Computer Methods in Applied Mechanics and Engineering. 95 (1992) 221-242.

[36] E.N. Dvorkin, K.J. Bathe, A continuum mechanics based four-node shell element for general nonlinear analysis., Engineering Computation 1 (1984) 77-88. 